\title{
BROOKHGWEN
}

NATIONAL LABORATORY

BNL-81630-2008-CP

\section{Sigma: Web Retrieval Interface for Nuclear Reaction Data}

\author{
B.Pritychenko, A.A.Sonzogni \\ 1 National Nuclear Data Center, Brookhaven National Laboratory, Upton, NY 11973, USA
}

Presented at the Covariance Workshop

Port Jefferson, New York,

June 24-27, 2008

\author{
National Nuclear Data Center \\ Energy Sciences \& Technology Department \\ Brookhaven National Laboratory \\ P.O. Box 5000 \\ Upton, NY 11973-5000 \\ www.bnl.gov
}

Notice: This manuscript has been co-authored by employees of Brookhaven Science Associates, LLC under Contract No. DE-AC02-98CH10886 with the U.S. Department of Energy. The publisher by accepting the manuscript for publication acknowledges that the United States Government retains a non-exclusive, paid-up, irrevocable, world-wide license to publish or reproduce the published form of this manuscript, or allow others to do so, for United States Government purposes. 


\section{DISCLAIMER}

This report was prepared as an account of work sponsored by an agency of the United States Government. Neither the United States Government nor any agency thereof, nor any of their employees, nor any of their contractors, subcontractors, or their employees, makes any warranty, express or implied, or assumes any legal liability or responsibility for the accuracy, completeness, or any third party's use or the results of such use of any information, apparatus, product, or process disclosed, or represents that its use would not infringe privately owned rights. Reference herein to any specific commercial product, process, or service by trade name, trademark, manufacturer, or otherwise, does not necessarily constitute or imply its endorsement, recommendation, or favoring by the United States Government or any agency thereof or its contractors or subcontractors. The views and opinions of authors expressed herein do not necessarily state or reflect those of the United States Government or any agency thereof. 


\title{
Sigma: Web Retrieval Interface for Nuclear Reaction Data
}

\author{
B. Pritychenko, ${ }^{*}$ A.A. Sonzogni \\ National Nuclear Data Center, Brookhaven National Laboratory, Upton, NY 11973-5000
}

(Dated: September 8, 2008)

\begin{abstract}
We present Sigma, a Web-rich application which provides user-friendly access in processing and plotting of the evaluated and experimental nuclear reaction data stored in the ENDF-6 and EXFOR formats. The main interface includes browsing using a periodic table and a directory tree, basic and advanced search capabilities, interactive plots of cross sections, angular distributions and spectra, comparisons between evaluated and experimental data, computations between different cross section sets. Interactive energy-angle, neutron cross section uncertainties plots and visualization of covariance matrices are under development. Sigma is publicly available at the National Nuclear Data Center website at www.nndc.bnl.gov/sigma.
\end{abstract}

\section{INTRODUCTION}

Motivated by the recent release of the ENDF/B-VII.0 evaluated nuclear reaction data library by CSEWG [1], the NNDC embarked on a project to develop an advanced Web interface for data dissemination, analysis and online processing of ENDF-6 formatted libraries. The resulting product, called Sigma would address the growing needs of traditional ENDF users as well as novel applications such as neutron cross section covariances. Sigma was designed focusing on achieving ease of use as well as taking advantage of the many software and hardware advances that took place in the last few years.

\section{WEB INTERFACE}

Sigma's web interface makes profuse use of modern HTML design, such as JavaScripts and CSS, as well as Java and relational database technologies. Sigma's primary goal is to provide search and browsing capabilities in a transparent way for four major evaluated libraries: ENDF/B-VII.0, JEFF-3.1, JENDL-3.3 and ENDF/BVI.8. Sigma should offer the raw ENDF-6 data as well as processed versions and plots. Additionally, Sigma should allow the comparison between evaluated data and experimental data from the EXFOR/CSISRS database, and basic mathematical operations between evaluated data.

The ENDF utility codes ENDF2HTML (ENDF Interpreted), X4toC4, ENDVER [2-4] and PREPRO [5], are used to process the raw ENDF data. PREPRO, in particular, is used to produce a point-wise version of the libraries for plotting and further computation. Plotting is performed by using the Java plotting package jplots [6].

Requests from users are processed by the 'Java Search \& Navigation Engine' that creates SQL queries. The results of the queries are stored in Java data structures and passed back to the user, or further processed with

\footnotetext{
*Electronic address: pritychenko@bnl.gov
}

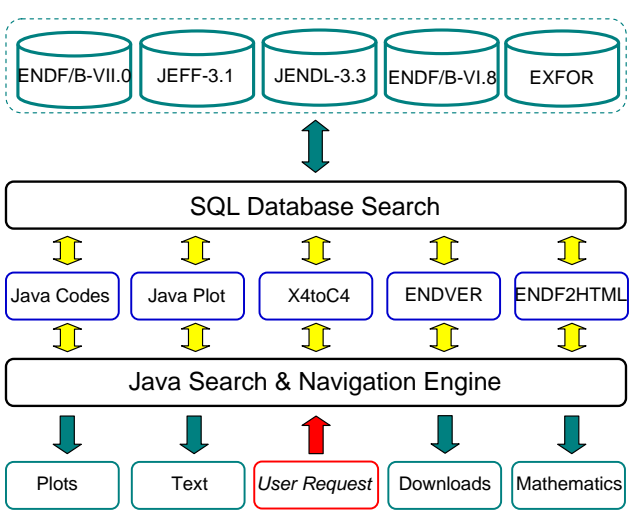

FIG. 1: Sigma Web Interface schematical design diagram.

jplots, ENDF2HTML, X4toC4 or ENDVER packages before making them available. Dedicated Java wrapping classes run FORTRAN utility codes for data processing and pass results to NNDC graphic package [6] for plotting. Final results are available in text, plot (in Portable Network Graphics format PNG) and zip formats. The Sigma schematical design diagram is shown in Fig. 1.

The Sybase ASE 15 relational database software is used in Sigma; a separate EXFOR database is available if necessary [7]. Relational design allows fast and flexible retrievals of evaluated and associated experimental nuclear reaction data; the main part of Sigma's database schema is shown in Fig. 2, it consists of four major tables: Evals, PlotTable, DataTable and X4toC4, as well as five additional tables. The original evaluated nuclear reaction data are stored in the Evals and DataTable, PlotTable contains pre-processed point-wise data and $\mathrm{X} 4$ toC4 contains pre-processed CSISRS/EXFOR data.

Sigma 1.0 was first made available to the public in April 2007, featuring browsing, searching and cross sections plots. Version 2.0 made its debut in January 2008, which incorporated angular distribution plots and mathematical operations of cross section data. Future work includes spectra plots, covariance visualization and vectorial graphics. Brief reviews of some of its most salient features are given in the following sections. 


\begin{tabular}{|l|}
\hline \multicolumn{1}{|c|}{ Evals } \\
\hline evalid int PK \\
nlib int \\
nver int \\
nsub int \\
mat int \\
z int \\
a int \\
created char(20) \\
authors char(50) \\
lab char(20) \\
elis float \\
liso int \\
zsymam char(11) \\
isnucleus char \\
iscompound char \\
iselement char \\
nlibid int \\
\hline
\end{tabular}

\begin{tabular}{|l|}
\hline \multicolumn{1}{|c|}{ PlotTable } \\
\hline ord int PK \\
\hline evalid int \\
mf int \\
mt int \\
x float \\
y float \\
\hline
\end{tabular}

\begin{tabular}{|l|}
\hline \multicolumn{1}{|c|}{ X4toC4 } \\
\hline ord int PK \\
projectile char(64) \\
target char(64) \\
charge int \\
atomic int \\
neutron int \\
mf int \\
mt int \\
energy char(128) \\
intenergy int \\
author char(128) \\
dataset char(64) \\
linetext char(256) \\
\hline
\end{tabular}

FIG. 2: Simplified Sigma relational database schema.

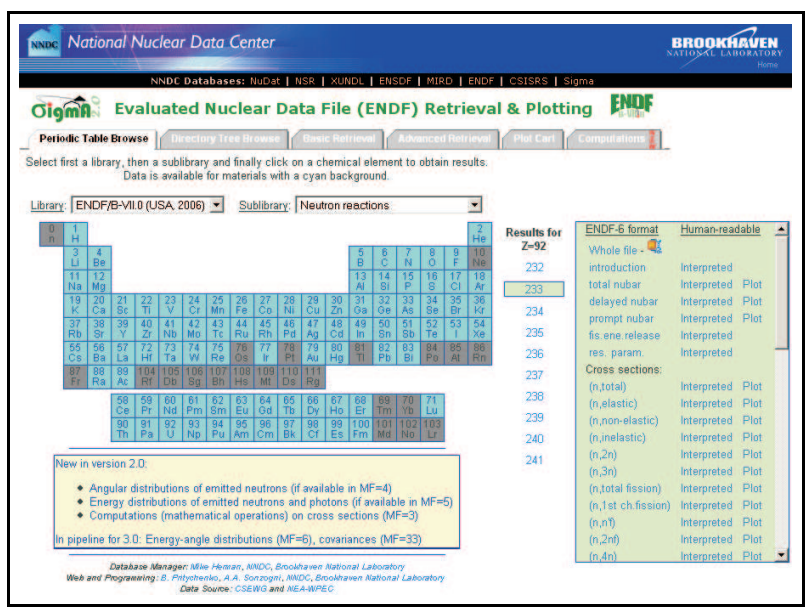

FIG. 3: Example of Sigma front page navigation capabilities (www.nndc.bnl.gov/sigma) for ${ }^{233} \mathrm{U}$.

\section{A. Browsing and Searching}

Sigma's front page has a 'Periodic Table' and 'Directory Tree' options for graphical navigation; on top of the 'Periodic Table' tab, there are pull-down menus to select library and sub-library. Fig. 3 shows an example of what the front page would look like after clicking on Uranium ( $\mathrm{U}, \mathrm{Z}=92$ ) and then clicking on $\mathrm{A}=233$. The scrollable table on the right of Fig. 3 with a light green background shows all the data available for this material, which can be retrieved in the ENDF-6 format, and some of them processed in an interpreted form or in a plot. Two search capabilities have been implemented, a basic one and a more advanced one. We have tried to shield the user from having to know the intricacies of the ENDF-6 format when using Sigma. However, the advanced search feature, would require some knowledge in order to make full use of it.

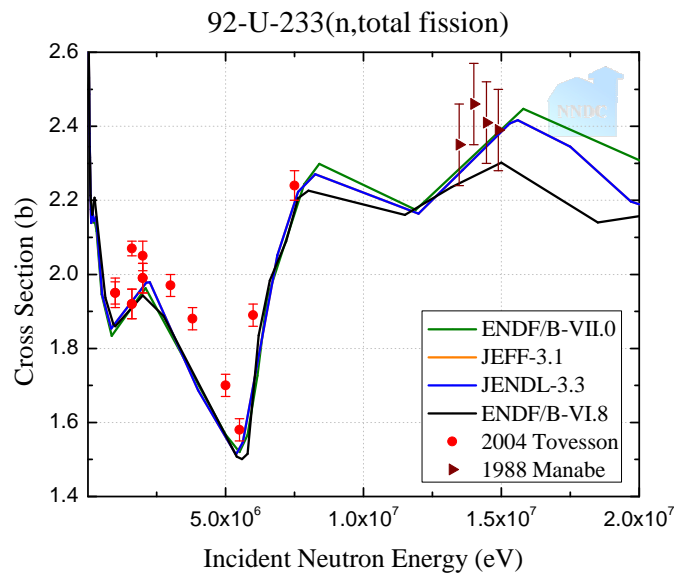

FIG. 4: Comparison between ENDF/B-VII.0, JEFF-3.1, JENDL-3.3 and ENDF/B-VI.8 libraries and selected experimental cross sections [8, 9] for ${ }^{233} \mathrm{U}$ (n,fission) reaction in the $0.1-20 \mathrm{MeV}$ neutron energy range.

\section{B. Connection to CSISRS/EXFOR}

A key feature in Sigma, and perhaps the most rewarding from the user's standpoint, is the possibility to also query the CSISRS/EXFOR database, which contains a wealth of experimental data that has been carefully added to the database over several decades. The connection is made possible by the fact that both Sigma and CSISRS/EXFOR use the same relational database software and by the work performed by V. Zerkin et al. [7] to facilitate this interconnection. The code X4toC4 [3] is used in Sigma to present data from CSISRS/EXFOR. As an example, cross sections for ${ }^{233} \mathrm{U}$ (n,fission) from different evaluated libraries together with selected experimental data sets $[8,9]$ in the $0.1-20 \mathrm{MeV}$ neutron energy range are shown in Fig. 4. The energy scale has been zoomed to show extra detail in the MeV region and only a few of the many experimental datasets are shown.

\section{Angular Distributions and Energy Spectra}

Neutron angular distributions, $\mathrm{d} \sigma / \mathrm{d} \omega$, are reconstructed from the $\mathrm{MF}=4$ file using the $\mathrm{MF}=3$ cross section data at $\mathrm{T}=300^{\circ} \mathrm{K}$. Absolute differential cross sections for emitted neutrons are defined as follows [10]:

$\sigma(\mu, E)=\sigma_{s}(E) f(\mu, E) / 2 \pi$,

where $\sigma_{s}$ is the scattering cross section given in $\mathrm{MF}=3$, $\mu$ is cosine of the angle for scattered neutrons, $E$ is the incident energy and $f(\mu, E)$ is the normalized probability as given in the $\mathrm{MF}=4$ file.

Evaluated neutron angular distributions can be accessed and compared with the experimental nuclear reaction data stored in Sigma. As an example, ENDF/BVII.0 ${ }^{233} \mathrm{U}$ (n,elastic) neutron angular distributions nor- 
malized to unity are shown in Fig. 5. One can see that the shape, as a function of the incident neutron energy evolves from the isotropic distribution at low energies into forward peaking distributions at higher energies. The cumulative plot of all angular distributions of neutron elastic scattering provides a useful tool for a quick assessment of the quality of evaluation.

ENDF/B-VII.0 Angular Distribution of Neutrons: 92-U-233(n,elastic)

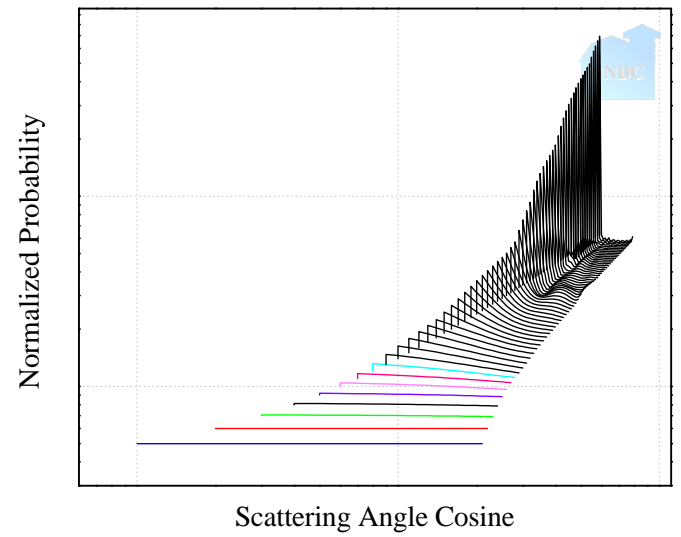

FIG. 5: ENDF/B-VII.0 ${ }^{233} \mathrm{U}$ (n,elastic) angular distributions normalized to unity for all incident neutron energies. A gradual shape evolution is indicative of a good evaluation quality.

Energy distribution, $\mathrm{d} \sigma / \mathrm{dE}$, plots are reconstructed from the $\mathrm{MF}=5$ file using the cross section data from $\mathrm{MF}=3$ at $\mathrm{T}=300^{\circ} \mathrm{K}[10]$ :

$d \sigma\left(E \rightarrow E^{\prime}\right) / d E^{\prime}=m \sigma(E) p\left(E \rightarrow E^{\prime}\right)$,

where $\sigma(E)$ is the cross section as given in $\mathrm{MF}=3, m$ is the neutron multiplicity and $p\left(E \rightarrow E^{\prime}\right)$ is the energy distribution from the $\mathrm{MF}=5$ file.

The plotting of energy-angle spectra $\left(\mathrm{d}^{2} \sigma / \mathrm{d} \omega \mathrm{dE}\right)$ is currently being tested. Reaction product (emitted neutrons, photons or residual nuclei) cross sections are calculated as [10]

$\sigma_{i}\left(\mu, E, E^{\prime}\right)=\sigma(E) y_{i}(E) f_{i}\left(\mu, E, E^{\prime}\right) / 2 \pi$,

where $i$ denotes one particular product, $E$ is the incident energy, $E^{\prime}$ is the energy of the product emitted with cosine $\mu, \sigma(\mathrm{E})$ is the interaction cross section $(\mathrm{MF}=3), y_{i}$ is the product yield or multiplicity and $f_{i}\left(\mu, \mathrm{E}, \mathrm{E}^{\prime}\right)$ is the normalized distribution from the $\mathrm{MF}=6$ file. Neutron energy spectra from the ${ }^{233} \mathrm{U}(\mathrm{n}, 2 \mathrm{n})$ reaction retrieved from the ENDF/B-VII.0 library are shown in Fig. 6.

ENDF evaluations contain angular distributions of emitted neutrons, photons and residual nuclei for a particular reaction channel. In general, many reaction channels are open and combined spectra are observed in experiments, creating a challenge when comparison between evaluation and experimental results need to be done. This was solved by the ENDVER code [4] that is integrated in Sigma as 'Full Spectra'.

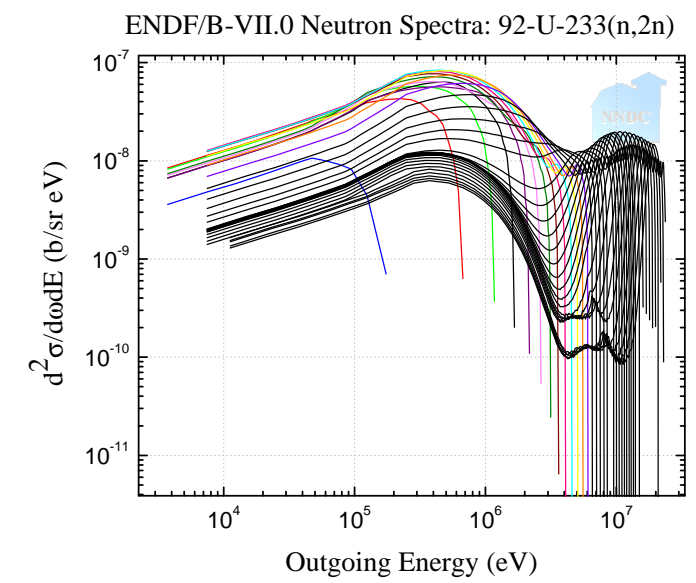

FIG. 6: Energy spectra of neutrons emitted from the ${ }^{233} \mathrm{U}(\mathrm{n}, 2 \mathrm{n})$ reaction at a $30^{\circ}$ scattering angle. The spectra for all neutron incident energies up to $30 \mathrm{MeV}$, contained in the ENDF/B-VII.0 file, are shown.

\section{Computations}

The possibility to perform mathematical operations with evaluated cross section data has been recently implemented. Sigma stores cross section data from $M F=3$ together with cross sections derived from the resonance parameters in $\mathrm{MF}=2$ that have been Doppler broadened to $300^{\circ} \mathrm{K}$ and linearized using PREPRO [5]. These data can be plotted simultaneously in Sigma for a variety of different reactions (MT values) using the Plot Cart feature. The Plot Cart is made up of different sets of $(\mathrm{x}, \mathrm{y})$ data points. Almost any mathematical equation can be easily typed in and a new set of $(\mathrm{x}, \mathrm{y})$ points resulting from such mathematical operation can be added to the Plot Cart and visualized.

As an example, the ENDF/B-VII.0 neutron capture cross section for the $s$-process nuclide ${ }^{100} \mathrm{Ru}$ is shown in Fig. 7, together with the same cross section multiplied by a factor of $E \times \exp (-E / 30000)$, a term proportional to a Maxwellian neutron flux distribution with $k T=30 \mathrm{keV}$. By examining the integrals of each curve, one can deduce that for this nuclide, the cross sections from the resonance region contribute the most of the Maxwellianweighted cross section at $k T=30 \mathrm{keV}$. We note that a recently released web application [11], offering Maxwellianaveraged cross sections and astrophysical reaction rates derived from ENDF-6 formatted evaluations, is a direct spin-off from Sigma.

Another example of mathematical operations would be the ratio of the ${ }^{237} \mathrm{~Np}$ (n,fission) from JENDL-3.3 and ENDF/B-VI.8, shown in Fig. 8. The disagreement between these two data sets of evaluated data in the fast region has triggered a recent experimental effort to accurately measure it [12]. These types of comparisons between data can be easily accomplished in Sigma. 


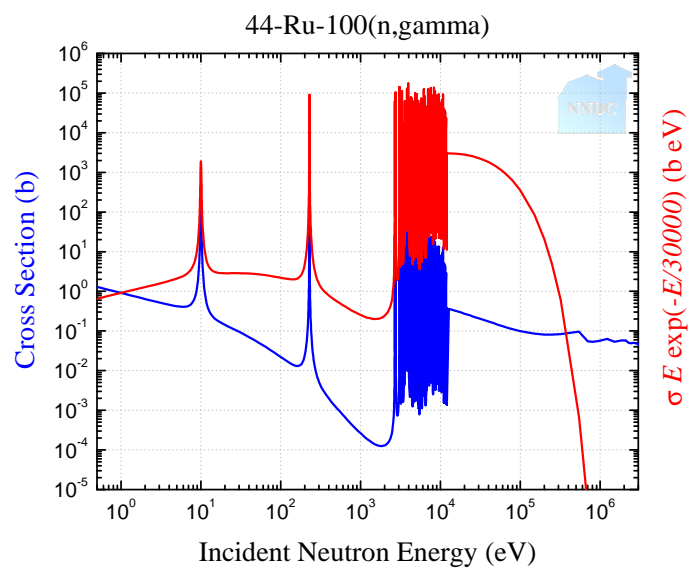

FIG. 7: Neutron capture cross sections for the $s$-process nuclide ${ }^{100} \mathrm{Ru}$ retrieved from ENDF/B-VII.0 (blue curve) and computed Maxwellian-weighted values for $k T=30 \mathrm{keV}$ (red).

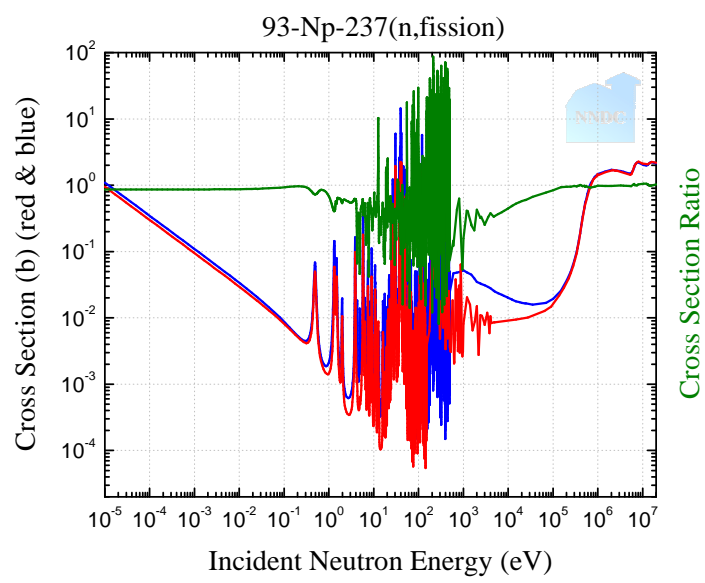

FIG. 8: Mathematical ratio of ${ }^{237} \mathrm{~Np}$ (n,fission) cross sections between ENDF/B-VI.8 and JENDL-3.3 libraries, green, red and blue curves, respectively.

\section{E. Cross Section Covariance Data}

The strong interest in the production and use of covariance data is evidenced by several recent sub-groups organized under the auspices of OECD-NEA as well as the current workshop. In response to this demand we are developing Sigma covariance capabilities. We have in mind a tool that would allow direct retrieval of covariance data and their visualization, which is important for both covariance producers and users. In this way we would avoid the use of complex processing codes, such as NJOY [13], where complete ENDF-6 files are needed and multigroup data must be produced before plot of uncertainties and correlations can be obtained.

In the ENDF-6 format cross section covariance matrices are stored in file $\mathrm{MF}=33$ and the resonance parameter covariance matrices in $\mathrm{MF}=32$. Retrieval of $\mathrm{MF}=33$ is straightforward, while $\mathrm{MF}=32$ would need pre-

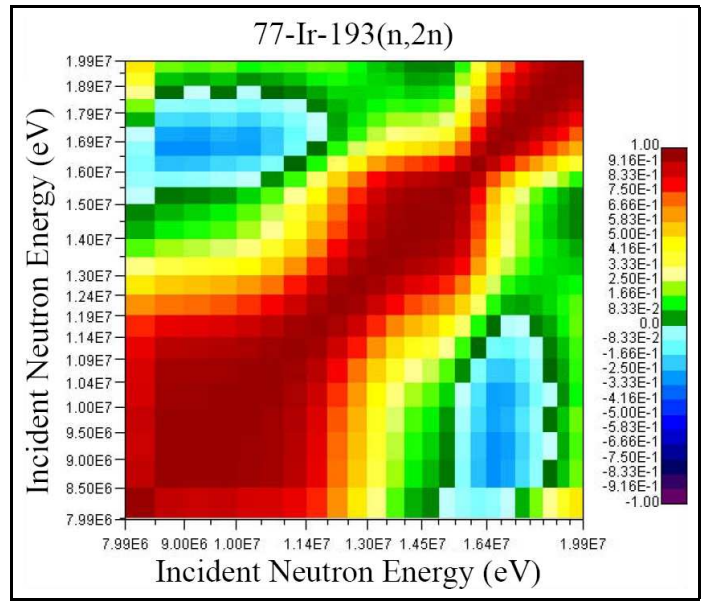

FIG. 9: ENDF/B-VII.0 correlation matrix for ${ }^{193} \operatorname{Ir}(\mathrm{n}, 2 \mathrm{n})$ reaction.

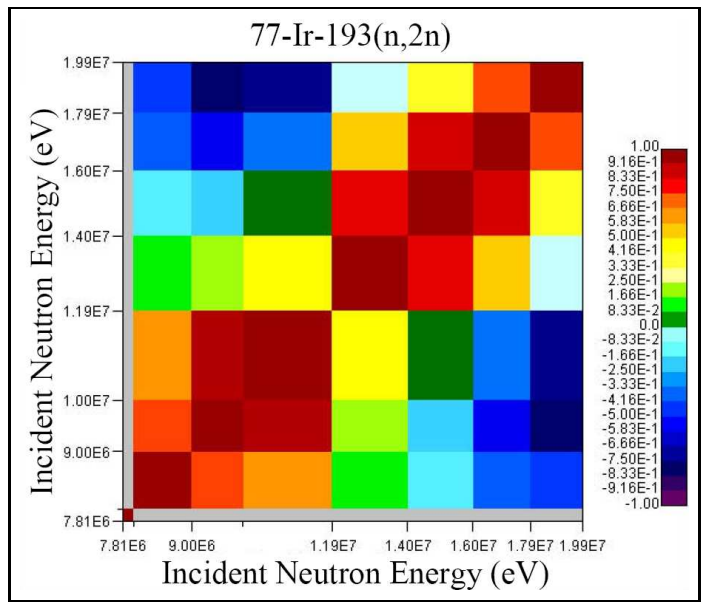

FIG. 10: The correlation matrix for ${ }^{193} \operatorname{Ir}(\mathrm{n}, 2 \mathrm{n})$ reaction from the low-fidelity project [14].

processing. In Fig. 9 we show an example of a correlation matrix directly retrieved from the ENDF/B-VII.0 library for the ${ }^{193} \operatorname{Ir}(\mathrm{n}, 2 \mathrm{n})$ reaction, which has a threshold of 7812 $\mathrm{keV}$.

Direct plotting of covariance matrices shows the data exactly as given in $\mathrm{MF}=33$, the disadvantage being that the covariance patterns are somewhat difficult to analyze and compare. Often, a more suitable approach is to visualize the same data as uncertainties and correlation matrices that are obtained after simple renormalization:

$\operatorname{corr}(i, j)=\frac{\operatorname{cov}(i, j)}{\sqrt{\operatorname{cov}(i, i) * \operatorname{cov}(j, j)}}$

In Fig. 10 we show the correlation matrix for ${ }^{193} \operatorname{Ir}(\mathrm{n}, 2 \mathrm{n})$ reaction produced by the Low-fidelity Project [14]. One can see a pattern with clearly visible strong positive correlations centered around the diagonal.

Sigma offers retrieval of covariances from the preloaded libraries, such as ENDF/B-VII.0 or Low-fidelity, but a 


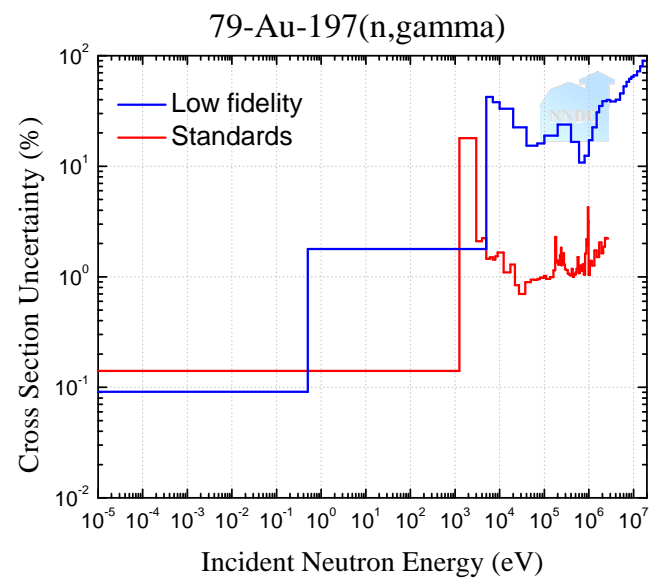

FIG. 11: Neutron cross section uncertainties for ${ }^{197} \mathrm{Au}(\mathrm{n}, \gamma)$ taken from the ENDF/B-VII.0 Standards sub-library are compared with the Low-fidelity data [14] .

user can also input a $\mathrm{MF}=33$ file and plot covariances. This would facilitate comparison of data, such as cross section uncertainties for ${ }^{197} \mathrm{Au}(\mathrm{n}, \gamma)$, where two extremes can be compared, the ENDF/B-VII.0 Standards sublibrary and the Low-fidelity Project [14], which are shown in Fig. 11.

\section{SUMMARY \& OUTLOOK}

Sigma Web interface, www.nndc.bnl.gov/sigma, provides a transparent and easy to use retrieval and visu- alization tool for the evaluated and experimental nuclear reaction data. Recent additions include energy spectra and angular distributions of emitted particles as well as mathematical operations of cross sections.

Sigma's covariance capabilities are currently being tested and will allow visualization of cross section covariance matrices directly retrieved from $\mathrm{MF}=33$ and, in the near future, also from $\mathrm{MF}=32$. Visualization of uncertainties and correlation matrices is straightforward and avoids the use of complex processing codes. Users will be able to retrieve and view covariance data from several preloaded libraries, including the Low-fidelity covariances, with a couple of clicks. In addition, users can input to Sigma their own data and view them as well. These new capabilities should not only facilitate covariance development, but should be appreciated also by covariance users.

Web access to evaluated and experimental nuclear reaction data continues to grow exponentially [15]. Continuing effort is needed to satisfy current needs and future demands.

\section{Acknowledgments}

We are grateful to M. Herman and A. Trkov for their help with ENDF utility codes, to P. Obložinský and M.T. Pigni for useful suggestions, and to M. Blennau and S.F. Mughabghab for a careful reading of the manuscript. This work was sponsored by the Office of Nuclear Physics, Office of Science of the U.S. Department of Energy under Contract No. DE-AC02-98CH10886 with Brookhaven Science Associates, LLC.
[1] M.B. Chadwick, P. Obložinský, M. Herman et al., Next Generation Evaluated Nuclear Data Library for Nuclear Science and Technology, Nucl. Data Sheets 107 (2006) 2931.

[2] R.E. MacFarlane, ENDF Interpreted (ENDF2HTML), Available from $\langle$ http://t2.lanl.gov/ $\rangle$.

[3] D.E. Cullen, Program $\mathrm{X}_{4} \mathrm{toC}_{4}$ : converts nuclear data from EXFOR format to a computational format, IAEANDS-80, September 1986.

[4] A. Trkov, ENDVER: ENDF file verification support package, Available from 〈http://www-nds.iaea.org/ ndspub/endf/endver/ $\rangle$.

[5] D.E. Cullen, The ENDF/B Pre-processing codes (PREPRO), Available from 〈http://www-nds.iaea.org/ ndspub/endf/prepro/ $>$.

[6] A.A. Sonzogni, private communication.

[7] V.V. Zerkin et al., EXFOR-CINDA-ENDF: Migration of Databases to Give Higher-Quality Nuclear Data Services, Inter. Conf. on Nucl. Data for Sci. and Tech., AIP $\mathbf{7 6 9}$ (2005) 586.

[8] F. Tovesson et al., 233Pa $(n, f)$ cross section up to En = 8.5 MeV, Nucl.Phys. A 733, 3 (2004).

[9] F. Manabe et al., Measurements of Neutron Induced
Fission Cross Section Ratios of 232Th, 233U, $234 U$, 236U, 238U, 237Np, 242Pu and 243Am Relative to $235 \mathrm{U}$ around $14 \mathrm{MeV}$, Tech. Rep. Tohuku University, 52, No.2.

[10] M. Herman (editor), ENDF-6 Formats Manual: Data Formats and Procedures for the Evaluated Nuclear Data File ENDF/B-VI and ENDF/B-VII, Tech. Rep. BNLNCS-44945-05-Rev, Document ENDF-102, Brookhaven National Lab, June 2005.

[11] B. Pritychenko, A.A. Sonzogni, Maxwellian-Averaged Cross Sections and Astrophysical Reaction Rates, Available from 〈http://www.nndc.bnl.gov/astro/ $\rangle$.

[12] F. Tovesson, T.S. Hill, Neutron-induced fission cross section of $237 \mathrm{~Np}$ from $100 \mathrm{keV}$ to $200 \mathrm{MeV}$, Phys. Rev. C 75, 034610 (2007).

[13] R.E. MacFarlane and D.W. Muir, The NJOY nuclear data processing system, version 91, Tech. Rep. LA-12740M, Los Alamos National Lab, NM, 1994.

[14] M.T. Pigni, M.Herman, P. Obložinský et al., Low-fidelity Covariance Project, these proceedings.

[15] B. Pritychenko et al., Nuclear Reaction and Structure Data Services of the National Nuclear Data Center, Ann. Nucl. Energy 33 (2006) 390. 